\title{
Robots Sing the Body Electric: Investigations of Body Language for Social and Spatial Interaction
}

\author{
Jaap Ham • Vanessa Evers • Manja Lohse
}

Published online: 12 July 2013

(C) Springer Science+Business Media Dordrecht 2013

Research in social robotics is thought to concern a future society where people and robots communicate socially. Robots are expected to interpret people's behaviors as well as generate meaningful social behaviors in order to seamlessly integrate into the inherently social fabric of human society. The current special issue focuses on the associations between robots and humans and aims to foster discussion on the development of computational models, robotic embodiments, and behaviors that enable robots to act socially. It presents novel research on the impact that robots have on people and their social and physical environment.

The investigation of associations between people and robots requires social interpretation when robot gestures and body movements influence ascription of anthropomorphism and likeability of robots, as is the case for the first paper of this special issue "To Err is Human(-like): Effects of Robot Gesture on Perceived Anthropomorphism and Likeability" by Maha Salem, Friederike Eyssel, Katharina Rohlfing, Stefan Kopp, and Frank Joublin. The authors present evidence suggesting that non-verbal behaviors displayed by humanoid robots affect the degree to which the robots are anthropomorphized and mental models are formed during interaction.

The paper "Interpretation of Emotional Body Language Displayed by a Humanoid Robot: A Case Study with Children”, by Aryel Beck, Lola, Cañamero, Antoine Hiolle, Luisa Damiano, Piero Cosi, Fabio Tesser, and Giacomo

\section{J. Ham $(\bowtie)$}

Human-Technology Interaction, Eindhoven University of Technology, Room IPO 1.36, PO Box 513, 5600 MB Eindhoven, The Netherlands

e-mail: j.r.c.ham@tue.nl
Sommavilla investigates the way in which robot body language can effectively communicate emotions to children. The authors aim to give humanoid robots the capacity to express emotions using their body. In their study, they investigated children's ability to recognize the emotional body language displayed by a humanoid robot. Results suggest that robot body postures and head positions can effectively convey emotions in child-robot interaction.

Telepresence robots have the added complexity of displaying the telepresence robot's body language as well as that of the remote operator. The paper "Easy-Interface and Control of Tele-Education Robots", by Sang-Seok Yun, Munsang Kim, and Mun-Taek Choi, offers a novel technique to convey a remote teacher's subtle body movements with robot movements. Results of a pilot study suggest that the robot's effective physical representation of the human teacher increased students' interest in the robot, and improved the learning experience.

In an ongoing human-robot dialogue based on gestures and body language, it is imperative to interpret user responses to the robot's actions. The paper "Using Embodied Multimodal Fusion to Perform Supportive and Instructive Robot Roles in Human-Robot Interaction”, by Manuel Giuliani, and Alois Knoll, introduces embodied multimodal fusion, a new approach for processing data from the robot's input modalities. By combining user input from various modalities with a representation of the robot's own possible actions the system can inform the action selection mechanism. This results in a more integrated dialogue of physical movements for instance in a construction task.

Human communication is not only social by nature but also inherently spatial. Effective social encounters require constant monitoring of and adjustment to spatial factors such as the gestures and movements of the interaction partner, proximity of others and the spatial context of the environ- 
ment. The paper "Design of a Parametric Model of Personal Space for Robotic Social Navigation”, by Elena Torta, Raymond H. Cuijpers, and James F. Juola, concerns the way in which robots and people spatially position themselves toward each other during interactions. They carried out experiments that suggested that human-human interaction distances are shorter than human-robot interaction distances during a communicative exchange, at least for the small humanoid robot that they used. Based on these experiments, a parametric personal space model for robotic social navigation was developed.

The paper "Automated Proxemic Feature Extraction and Behavior Recognition: Applications in Human-Robot Interaction”, by Ross Mead, Amin Atrash, and Maja J. Matarić investigates the physical and psychophysical factors that determine social spacing between people and robots. Their results demonstrate that Hidden Markov Models trained on psychophysical features outperform those trained on physical features. This results in a powerful representation of proxemic behavior relevant for socially interactive and assistive robots.

The paper "BEHAVE-II: A Revised Set of Measures to Assess Users's Attitudinal and Behavioral Responses to a Robot's Social Behaviors", by Michiel Joosse, Aziez Sardar, Manja Lohse, and Vanessa Evers describes the development of a data collection instrument to assess people's responses towards robot's gestures and body movements. The paper bridges the previous papers by testing the validity of BEHAVE-II in a proximity-related experiment where a robot invaded the personal space of participants.

The desire to make robots easier to use explains why developers and designers of robots leverage the way people socially interact with each other. By creating social robot gestures and body movements that can be interpreted meaningfully in a social way, the robot is positioned as a social actor. This special issue contains two studies that investigate the effects of the robot as a social actor in real-world contexts. To explore the impact of the robot's 'body electric' from different perspectives, the studies explore robots that display only simple spatial movements and robots that combine verbal and non-verbal social interaction behaviors as well as laboratory and real-world settings.

The paper "Living with a Vacuum-Cleaning RobotA 6-month Ethnographic Study", by Julia Fink, Valérie Bauwens, Frédéric Kaplan, and Pierre Dillenbourg assesses the usage and social implications of long-term use of domestic service robots in people's homes. In actual homes of participants, the authors studied participants' perceptions of a vacuum cleaning robot, the changes of these perceptions over time, usage patterns, and social activities related to the robot. Analyses revealed several factors that promote or hinder the process of adopting a domestic service robots. Based on these findings, the authors make suggestions to further improve the design of functional home robots for long-term acceptance.

The paper “When a Robot's Group Membership Matters: Anthropomorphization of Robots as a Function of Social Categorization”, by Dieta Kuchenbrandt, Friederieke Eyssel, Simon Bobinger, and Maria Neufeld investigates the effect of positioning a robot as an in-group or non in-group member on anthropomorphization and acceptance. To assess anthropomorphization they used an innovative, implicit measurement procedure. Results suggested that when the robot is perceived to be an in-group member, participants anthropomorphize it more, and also evaluate it more positively compared with a robot as an out-group member.

The papers in this issue offer a combined overview of the interpretation of robot body language for social and spatial interaction, the design and assessment of gestural and body movement behaviors, the technical realization of body movements as well as the assessment in laboratory as well as real-world contexts. We therefore hope that this special issue offers the reader insights into the main challenges when designing and developing robot body-language for social and spatial human-robot interaction.

We would like to thank Editor in Chief Shuzhi Sam Ge and Associate Editor John-John Cabibihan from the Editorial Board, as well as Anand David and Nathalie Jacobs from Springer for their guidance and advice.

We very warmly want to thank our reviewers who set the high standards of this special issue: Kai Arras, Philip Baer, Paul Baxter, Christian Becker-Asano, Cindy L. Bethel, Laura Boccanfuso, Paul Bremner, Sonia Chernova, Joachim de Greeff, Louis-Philippe Demers, Shuonan Dong, Boris Duran, Christopher Ford, Barbara Gonsior, Frank Hegel, Dirk Holz, Myung-Suk Kim, Yungyung Kim, Alexandra Kirsch, Raj Madhavan, Ross Mead, Nicole Mirnig, Hirotaka Osawa, Amit Kumar Pandey, Bogdan Raducanu, Astrid M. Rosenthal-von der Pütten, Peter Ruijten, Maha Salem, Satoru Satake, Stefan Scherer, Candace Sidner, Adriana Tapus, Anna-Lisa Vollmer, Astrid Weiss, and Agnieszka Wykowska.

Guest Editors

Jaap Ham is Associate Professor in the Human-Technology Interaction research group at Eindhoven University of Technology. He studies the psychology of human-technology interaction, investigating social robotics, (ambient) persuasive technology, and trust and acceptance of technology.

Vanessa Evers is Professor of Human Media Interaction at the University of Twente. She is a leading scholar in Human Robot Social Interaction. She has a $\mathrm{PhD}$ from the Open University, UK and was a visiting Scholar at Stanford University. Previously employed by the University of Amsterdams Psychology and Computer Science 
department, Vanessa Evers works on social, cultural, emotional, and motivational aspects of interacting with intelligent agents such as robots.

Manja Lohse is a postdoctoral researcher at the Human Media Interaction group at University of Twente. Until June 2012 she was a member of the Research Institute for Cognition and Robotics (CoR-Lab) in the Hybrid Society Group at Bielefeld University. Manja Lohse's research interests are the social aspects of human-robot interaction, evaluation methods, and users' expectations toward robots. 